Introduction/Background* The high-dose-rate intra-cavity brachytherapy (HDR-ICBT) has an important and essential role for radiation therapy for uterus cervical cancer ad rectal dose reduction is critical for determining the radiation dose for HDR-ICBT. In Asia, standard external beam radiation therapy combines whole pelvic radiation therapy (WPRT) and sequential WPRT with central shielding (WPRT-CS) for rectal dose reduction prior to HDR-ICBT. This study aimed to evaluate the treatment outcome and tolerance of the combination treatment strategy.

Methodology Forty-six cervical cancer patients treated from 8/ 2011 to 9/2020 were included in this retrospective analysis. All patients received combination therapy of HDR-ICBT and EBRT which is consisted of WPRT and WPRT-CS using IMRT/TomoTherapy.

Result(s)* Primary tumor stages (FIGO) were distributed as follows: IB: 6, IIA: 4, IIB: 12, IIIA: 1, IIIB: 3, IIIC1:17, IIIC2:3 (with Para-aortic lymph node metastasis), pelvic lymph node metastasis $(\mathrm{N}+)$ : 21. Forty patients had squamous cell carcinoma (SCC) and the remaining six had adenocarcinoma. The median age of patients was 65.5 (range, 30-91) years. All patients completed treatment, and the median dose of WPRT and WPRT-CS was 36 (range, 20-43.2) and 14.4 (range, 7.228) Gy, respectively. Median HDR-ICBT dose/fractions to Point A was 25 Gy/5 Fr (range, 15-30 Gy/3-5 Fr). Thirty-five had weekly cisplatin concomitant with RT.

Median follow-up was 43.5 (range, 4-122) months, and 5 -year local control, disease-free survival, and overall survival rates (OS) were 95.6\%, 84.0\%, and 83.1\%, respectively. There was only two local failure and seven patients experienced distant metastasis (4 lung metastasis and 3 para-aortic metastasis). Four of seven patients with distant metastasis received salvage therapy and the disease got controlled. One patient who controlled cervical cancer, developed primary lung cancer and died of that. No acute or late $\geq$ Grade 3 radiation-induced genitourinary or gastrointestinal toxicities were observed.

Conclusion* In the present study, the combination of IMRT consisting of WPRT and WPRT-CS using TomoTherapy and HDR-ICBT show comparatively long-term great local disease control. and tolerance rates for cervical cancer patients. This combination strategy is considered one of the effective and safe treatment options for cervical cancer.

\section{FALSE NEGATIVE RATE AT 18F-FDG PET/CT INPARA- AORTIC LYMPHNODE INVOLVEMENT INPATIENTS WITH LOCALLY ADVANCED CERVICALCANCER: IMPACT OF PET TECHNOLOGY}

S Gouy*, V Seebacher, C Chargari, M Terroir, A Maulard, C Genestie, P Pautier, A Leary, P Morice, D Deandreis. Gustave Roussy, Surgery, Villejuif

\subsection{6/ijgc-2021-ESGO.87}

Introduction/Background* The identification of factors responsible for false negative $(\mathrm{FN})$ rate at $18 \mathrm{~F}$ - Fluorodeoxyglucose (FDG) Positron Emission Tomography/Computed Tomography (PET/CT) in para-aortic (PA) lymph nodes in the presurgical staging of patients with locally advanced cervical cancer (LACC) is challenging. The aim of this study was to evaluate the impact of PET/CT technology.

Methodology A total of 240 consecutive patients with LACC (International Federation of Gynecology and Obstetrics, FIGO, stage IB2-IVA) and negative Magnetic Resonance Imaging (MRI) and/or Computed Tomography (CT) and negative 18FFDG PET/CT in the PA region, undergoing laparoscopic PA lymphadenectomy before chemoradiotherapy were included. The FN rate in patients studied with Time of flight (TOF) PET/CT (TOF PET) or non-Time of flight PET/CT (no-TOF PET) technology was retrospectively compared.

Result(s)* Patients presented with FIGO stage IB ( $\mathrm{n}=78)$, stage IIA-B $(\mathrm{n}=134)$, stage III $(\mathrm{n}=18)$ and stage IVa $(\mathrm{n}=$ $10)$, squamous cell carcinoma $(\mathrm{n}=191)$ and adenocarcinoma ( $\mathrm{n}=49$ ). 141/240 patients were evaluated with no-TOF PET/ CT and 99/240 with TOF PET/CT. Twenty-two patients (9\%) had PA nodal involvement at histological analysis and considered PET/CT FN findings. The FN rate was $8.5 \%$ for noTOF PET and $10 \%$ for TOF PET subgroup respectively ( $\mathrm{p}=$ 0.98). Ninety patients (38\%) presented with pelvic node uptakes at PET/CT. The FN rate in the PA region was $18 \%$ $(16 / 90)$ and $4 \%(6 / 150)$ in patients with and without pelvic node involvement at PET/CT respectively (19 vs 3\% for noTOF PET and 17 vs 5\% for TOF PET subgroup).

Conclusion* In LACC, FN rate in PA lymph nodes detection is a clinical issue even for modern PET/CT, especially in patients with pelvic uptake. Surgical lymphadenectomy should be performed in case of negative PET/CT at PA level in these patients, while it could be discussed in the absence of pelvic uptake.

\section{A NEW SURGICAL ERA FOLLOWING THE LACC TRIAL - A TEN STEP SURGICAL APPROACH TO FERTILITY SPARING ABDOMINAL RADICAL TRACHELECTOMY}

S Smyth, H Jiang* , C Pappa, M Abdalla, H Soleymani. John Radcliffe Hospital, UK

\subsection{6/ijgc-2021-ESG0.88}

Introduction/Background* Minimally invasive surgery in the management of cervical cancer was dealt a heavy blow in 2018, with the laparoscopic approach to cervical cancer trial (LACC) taking the gynaecology oncology world by storm; leading to a complete overhaul of surgical techniques involved.

Methodology We present a 34-year-old fit and well nulliparous female referred with grade two squamous cell carcinoma; $4.3 \mathrm{~mm}$ invasion depth on loop histology, stage 1A2. There was no radiological evidence of residual tumour, lymphadenopathy or metastatic disease. Following counsel, the patient underwent midline laparotomy, bilateral pelvic lymph node dissection and radical trachelectomy with no residual and 37 lymph nodes negative and uncomplicated recovery.

Result(s)* We present a stepwise surgical approach:

1. Midline laparotomy and pelvic side wall opening to develop eight avascular retroperitoneal spaces of pelvis with slinging of round ligaments and ureters and preservation of fallopian tubes and ovaries

2. Bilateral pelvic lymph node dissection sent for frozen section examination

3. Full mobilisation of cervix with ureterolysis, dissection of parametrium, paracolpium and vaginal cuff. Skeletonisation to bifurcation, sacrifice and preservation of descending and ascending uterine artery branches respectively - ensuring artery pulsation and uterine body perfusion during procedure

4. Measurement of the vaginal cuff from the central tumour bed - identification of a $2 \mathrm{~cm}$ clear margin 